index ecomunicación | no 11(2) 2021 | Páginas 109-132

E-ISSN: 2174-1859 | ISSN: 2444-3239 | Depósito Legal: M-19965-2015

Recibido el 17_01_2021 | Aceptado el 18_05_2021 | Publicado el 15_07_2021

\title{
VIDEOJUEGOS DE MUNDO ABIERTO: PROPUESTA DE UN MODELO DE ANÁLISIS LUDONARRATIVO
}

OPEN-WORLD VIDEOGAMES:

A LUDONARRATIVE MODEL PROPOSAL

https://doi.org/10.33732/ixc/11/02Videoj

Rubén García Moreno

Universidad de Valladolid

garmo.ruben@gmail.com

https://orcid.org/0000-0001-9932-0933

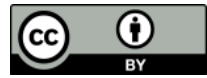

Para citar este trabajo: García Moreno, R. (2021). Videojuegos de mundo abierto: propuesta de un modelo de análisis ludonarrativo.

index.comunicación, 11(2), 109-132.

https://doi.org/10.33732/ixc/11/02Videoj 
Resumen: Los videojuegos de mundo abierto se han popularizado en los últimos años erigiéndose como una nueva forma de diseñar experiencias de juego, ofreciendo nuevas técnicas narrativas, alternativa a otras lineales más tradicionales. Su proposición lúdica está basada en amplios espacios transitables, poblados por personajes controlados por inteligencia artificial y un uso intensivo de la narración ambiental que permite a sus creadores explorar nuevas formas narrativas. El presente trabajo tiene como objetivo proponer un modelo de análisis para el estudio académico de videojuegos de mundo abierto, tomándolos en consideración como construcciones ludonarrativas. El trabajo se fundamenta con teorías científicas de los primeros académicos que se han aproximado a este tipo de videojuegos, pero también con base en la visión de los creadores que han dedicado parte de su carrera a diseñarlos. El trabajo, por último, se completa con una puesta en práctica del modelo de análisis sobre Death Stranding, videojuego de mundo abierto, y una discusión de los resultados.

Palabras clave: videojuegos; mundo abierto; narrativa; narración ambiental; diseño de videojuegos.

Abstract: Open-world videogames have become popular in the last years, establishing themselves as a modern way of designing videogame experiences as they offer new narrative techniques, alternative to other traditional linear ones. Its ludic proposal is based on wide passable spaces, inhabited by characters controlled by artificial intelligence, and an intensive use of environmental narration that allows its creators to explore new narrative forms. The present work aims at proposing a new model of analysis for the scientific study of open-world videogames, considering them as ludonarrative constructions. This work is grounded on scientific theories of the first academic researchers that have approached these types of videogames, but it is also based on the vision from the creators who have dedicated part of their career to designing them. Lastly, this work is completed with an implementation of the analysis model on Death Stranding, an open-world videogame, and a discussion of the results.

Keywords: Videogames; Open-World; Narrative; Environmental Storytelling; Game Design. 


\section{Introducción y objetivos}

Los videojuegos han evolucionado a lo largo de sus décadas de existencia y, con ellos, la forma en que se comunican con el jugador. Se trata de experiencias audiovisuales interactivas que se componen de aspectos propios del juego (Frasca, 1999), así como técnicas narrativas heredadas de medios audiovisuales y escritos (Laurel, 2013; Murray, 1999) que han adquirido singularidades propias (Aranda, Gómez, Navarro-Remesal y Planells, 2015).

$\mathrm{Su}$ desarrollo ha ido de la mano de las innovaciones tecnológicas, aprovechando las posibilidades de las nuevas generaciones de equipos y consolas. Esta evolución se ha visto reflejada en propuestas novedosas e innovadoras tanto en el plano de juego como en la narración, y uno de los modelos más extendido de los últimos años ha sido el de los mundos abiertos. Aunque existían propuestas puntuales previas, Grand Theft Auto III (DMADesign, 2001) consolidó un nuevo paradigma en el diseño, muy orientado hacia la libre exploración y experimentación en un gran entorno tridimensional (Garrelts, 2006; Solo, 2019). Desde aquel año 2001 hasta el presente, el modelo se ha perpetuado a lo largo de los años, permitiendo nuevas formas de jugar (Muncy, 2015) y de contar historias.

La investigación científica en la parcela concreta de los videojuegos de mundo abierto se encuentra en una fase temprana en la que han surgido estudios de caso (Pérez, 2015) pero no se ha teorizado en profundidad sobre sus principios de diseño. Por ello, esta investigación ofrece una aproximación que conjuga tanto el planteamiento académico de los game studies como la visión más técnica de autores veteranos en la industria para conseguir un acercamiento lo más útil posible, de cara a su empleo práctico en investigaciones futuras.

El objetivo de este trabajo es proponer un modelo de análisis de videojuegos de mundo abierto que sirva para articular estudios académicos de este modelo de títulos. Los interrogantes planteados por este modelo identificarán, de forma exploratoria, el diseño de la experiencia de juego, la propuesta narrativa, la construcción de mundos o el comportamiento de la inteligencia artificial, entre otros aspectos, a partir de las premisas teóricas anteriores y la observación sistemática (Kelle, 2010). En los últimos apartados de este trabajo se ofrecerán los resultados obtenidos tras aplicar el modelo de análisis a Death Stranding (Kojima-Productions, 2019), un popular videojuego de mundo abierto. 


\section{Teorías en torno a los videojuegos de mundo abierto}

El diseño de videojuegos de mundo abierto presenta algunas particularidades que han sido apuntadas por algunos trabajos académicos, aunque han recibido una mayor amplitud por parte de los propios diseñadores en conferencias y análisis post-mortem. La novedad de este análisis recoge ambos tipos de aportaciones para desarrollar un marco teórico, que se utilizará para construir un modelo de análisis aplicado a los videojuegos de mundo abierto. Su estructura seguirá los preceptos básicos de diseño de videojuegos, es decir, la dualidad de experiencias, lúdica y narrativa, seguida de apartados más específicos para los mundos abiertos como la configuración del mundo y los sistemas.

\subsection{Diseño de la experiencia de juego}

\subsubsection{Objetivo y tema}

Esta investigación parte de la teoría de Chis Crawford (1984: 49-50), al establecer dos conceptos básicos para el diseño de videojuegos: el objetivo y el tema. Al definir un objetivo, el diseñador establece qué fantasías y emociones pretende que sienta el jugador; mientras que un tema sirve para dar forma al entorno en el que se experimentará el juego, a la vez que permite dar forma al objetivo. Siguiendo ejemplos del autor de la teoría, el objetivo podría ser ejercer el liderazgo y, el tema, la leyenda del Rey Arturo.

A partir de esta premisa, las mecánicas de juego sirven para que el jugador interactúe con el objetivo y el tema establecidos en la etapa de diseño. Estas mecánicas son las actividades esenciales que repite a lo largo de la partida (Parker, 2015: 4; Zimmerman \& Salen, 2003: 316), pudiendo ser una única acción o un conjunto de ellas. Estas mecánicas se expresan a través de un conjunto de "paleta de acciones disponible para el jugador» (Crawford, 2005: 91-110) y actúan como vehículos conductores de la comunicación entre el jugador y el creador: en primer lugar, permiten que el primero cumpla los objetivos de juego que le son propuestos y, en segundo, sirven para que la narración fluya (Tremblay, 2020).

En ese sentido, Sicart (2008) plantea tres tipos de mecánicas de juego. Las primeras serían las mecánicas primarias, que son aquellas que se emplean directamente para la superación de desafíos, por ejemplo, apuntar y disparar en un juego de acción. Siguiéndolas en importancia, el autor indica que las mecánicas secundarias son aquellas que ayudan a afrontar los desafíos y son ejecutadas frecuentemente, pero no sería posible superarlos exclusivamente a través de ellas. Siguiendo el mismo ejemplo de un juego de acción, el salto puede superar al jugador a esquivar los disparos o plantear determinadas estrategias, pero no puede acabar con los enemigos de esta forma. Por último, se reconoce 
un tercer conjunto de mecánicas, aquellas que no están implementadas en el videojuego con el objetivo de superar los objetivos.

\subsubsection{Libertad y autonomía del jugador}

Una de las principales premisas del diseño de los videojuegos de mundo abierto es la libertad y autonomía del jugador (Min, Mott, Rowe, Liu y Lester, 2016; Shroff, 2014). Se encuentran orientados hacia un desempeño del jugador que le permita establecer sus propios objetivos (Squire, 2008) y que se traduce en diferentes tipos de actuación, elegidos con libertad por parte del jugador, en su experiencia de juego. En primer lugar, se le otorga una plena facultad de desplazamiento por el mundo, ya que uno de los objetivos de este diseño es que el jugador mantenga una exploración activa del terreno (Bergeron, 2017). Por otro lado, la libertad le permite planificar su partida, eligiendo los caminos y experiencia a descubrir en su sesión (Min et al., 2016).

La autonomía del jugador va acompañada necesariamente de otro concepto clave en el diseño de estos videojuegos: una partida no estructurada (Claussen, 2019). El diseño de los mundos abiertos a menudo emplea estrategias de juego propias de videojuegos sandbox (Canary, 2014; Shroff, 2014), en los que prima la falta de linealidad y la experimentación (Bermejo, 2013; Mikkelsen \& Podenphant, 2019). La partida de un título definido como sandbox ofrece libertad de secuenciación de los desafíos por parte del jugador, en lugar de establecer un orden fijo. Esto a menudo significa que se le plantean múltiples retos, y estos no pueden ser superados simultáneamente, sino que, por el contrario, es tarea del jugador establecer un orden.

Por otra parte, en estos juegos también se le propone al jugador un espacio de experimentación en el que tomar decisiones no establecidas previamente, no conocidas por el autor (Walton \& Suckling, 2017). Le proveen de diversas herramientas y un objetivo, o varios, y recae del lado del jugador emplear un camino resolutivo entre todos los posibles. Estas características descritas que se asocian al diseño de partida sandbox son igualmente reconocibles en los videojuegos de mundo abierto a pesar de que, como se ha señalado, se trate de una «libertad dirigida» (Navarro, 2013).

\subsection{Diseño de la experiencia narrativa}

\subsubsection{Historia de autor e historia del jugador}

Algunos creadores de videojuegos subrayan que los videojuegos de mundo abierto, al dejar espacio para la libertad de actuación y la expresión creativa del jugador, plantean una narrativa que funciona a dos niveles. A menudo cuentan con un «modo historia» o «historia principal», es decir, el guion de acontecimientos que 
son impuestos por una figura que, en cuanto a autoridad, está en una posición superior: el creador (Van-Lierop, 2018). Esta historia no emerge de la partida al interactuar el jugador con los demás elementos del diseño, sino que tiene un desarrollo predefinido y pautado y, por tanto, siempre tiene lugar, aunque en ocasiones pueda dejar espacio a una toma de decisiones encauzadas.

Por otro lado, cuando el jugador no juega sin cumplir los objetivos propuestos, está escribiendo su propio relato (Nelson, 2018). Esta historia del jugador es la que mayor libertad expresiva permite, y se compone de las situaciones que este construye mientras interactúa con los sistemas y con el mundo (Van-Lierop, 2018).

La particularidad de esta técnica narrativa consiste en que ambas historias, la del autor y la del jugador, las protagoniza el mismo personaje (Isbister, 2017). De esta forma, el personaje protagonista cumple dos funciones diferenciadas según el momento de la partida: personaje instrumental o personaje dramático (Cuadrado, 2013). Primordialmente, el personaje cumple la función de avatar del jugador, puesto que sirve como representación corpórea de los comandos ejecutados y el resultado de sus acciones. Sin embargo, en otros compases puede ocurrir que se le atribuyan las características del personaje cinematográfico, teatral o literario y entonces se le asocian «emoción, psicología, rasgos, etc.» (Cuadrado, 2013: 163)

Algunos diseñadores advierten que las situaciones a las que se enfrenta el personaje protagonista deben mantener la coherencia. Un desafío complejo asumiendo la premisa de entregar libertad al jugador y favorecer su experimentación, debido a que en unas ocasiones se encontrará tomando parte en unas acciones prediseñadas, como son las misiones de la historia del autor, pero en otros momentos, cuando está teniendo lugar la historia del jugador, el mismo personaje podría estar construyendo un relato que no tuviera que ver con los objetivos de dicho personaje en la historia (Nelson, 2018).

En este sentido, los creadores han manifestado que el diseño narrativo de los mundos abiertos supone un desafío, ya que deben facilitar el acercamiento de ambas historias, construir una narrativa que difumine la línea entre ambos relatos para que tanto la historia como el juego sean una experiencia unitaria (Nelson, 2018).

\subsubsection{El funcionamiento del tiempo}

Los videojuegos presentan una representación propia en la representación de la temporalidad, diferente a otros medios (Juul, 2005), pero las particularidades en este aspecto del diseño son muchas (Fernández-Vara, 
2009; Navarro, 2013), ya que se aprecian variaciones en su representación según la obra.

En algunos casos el tiempo de la partida puede coincidir con el mundo real (Zagal \& Mateas, 2007), como ocurre en Animal Crossing (Nintendo-EAD, 2012), pero en otros la narración de la obra puede no coincidir con el contexto en el que la partida se está desarrollando. Por ello, se apunta a que el tiempo de juego gametime (Juul, 2005) puede estar unido al desempeño del jugador, siendo en tal caso céntrico, o excéntrico cuando está desvinculado de este (Pérez, 2010: 113).

Al observar la aplicación de la temporalidad en el diseño narrativo de los videojuegos de mundo abierto, se aprecia que es común la diferenciación entre discurso e historia enunciada por Todorov (2006) debido a su diseño ludonarrativo. La libertad del jugador, aunque dirigida (Navarro, 2013), favorece que los acontecimientos no se presenten siempre del mismo modo, especialmente si dedica tiempo a construir su propia historia, que es potencialmente única, en lugar de seguir la del autor. Debido a esta emergencia de los acontecimientos, los diseñadores afirman que este tipo de obras no son deterministas (England, 2016). Al permitir espacio para la variabilidad, el diseño de los acontecimientos posibles en el mundo de juego necesita que se conciban desligados de una temporalidad concreta, especialmente durante la historia del jugador.

La distinción entre discurso e historia puede verse al atender a la historia del autor y la del jugador por separado. De esta manera, la historia del autor se identificaría con el discurso en el sentido de que en ambos el tiempo es lineal. Presentan los acontecimientos de una forma secuencial, siguiendo una línea temporal fija. Por ejemplo, el jugador debe superar las misiones de juego en un orden establecido para completarlo.

Pero, por otra parte, la historia, identificada en estos juegos con la propia del jugador, se desvincula de la temporalidad, especialmente en los modelos excéntricos que propone Víctor Navarro. No necesitan un tiempo para existir, ya que puede tener lugar en cualquier momento, e incluso podrían estar sucediendo acontecimientos simultáneos que el mundo no necesita ordenar. De esta forma, en un mundo como el de Grand Theft Auto V (Rockstar-North, 2013), el jugador podría estar explorando un barrio en cualquier momento del día mientras los sistemas de juego han creado la situación emergente de una persecución policial entre personajes del mundo. 


\subsection{Mundo y simulación}

Los videojuegos pueden ser estudiados como mundos ludoficcionales (Planells, 2013), es decir, mundos narrativos interactivos en los que las reglas del juego se encuentran necesariamente relacionadas con el relato. Partiendo de esta premisa, el espacio de juego de los mundos abiertos pretende ser un entorno virtual que, a través de mecánicas de juego, la inserción de unos habitantes del lugar y otros mecanismos narrativos consigue una simulación verosímil de la vida.

La verosimilitud puede ser un desafío para el diseño de videojuegos. Por ello, los creadores se apoyan sobre recursos que han empleado el cine y la literatura, entre otras artes. En este sentido, la geógrafa Kate Edwards (2016) cree que el diseño de los mundos videolúdicos se beneficia de planificar el mundo a través de capas de contenido. Estas capas se corresponderían con la geografía, la física, la climatología, la biología, estructuras sociales, el idioma y todas aquellas materias tratadas por las ciencias naturales o humanas que aporten sentido al lugar. Todos estos elementos expresan la presencia de una cultura en el mundo de juego y consiguen construir el contexto narrativo de la propuesta ludoficcional.

Aparte de las capas relacionadas con ciencias del mundo real, también es relevante la historia del mundo que habita el avatar del jugador, ya que aporta mayor consistencia, al tiempo que expande el relato del autor. Se plantea un mundo que es -en sí mismo- un lienzo sobre el que pintar detalles de la narración a través del arte y el diseño de los escenarios, y que puede cumplir las mismas funciones narrativas que un personaje de la trama (Connel, 2017), como apuntan los diseñadores de Ghost of Tsushima (Sucker-Punch, 2020). En este sentido, Nitsche (2008: 3) apunta que el diseño del mundo contiene unos «elementos narrativos evocadores», que no cuentan una historia completa por sí mismos, pero sirven para dar comienzo al proceso narrativo en el imaginario del jugador. En algunos casos, el mundo de juego podría ser el principal recurso narrativo del juego (Walton \& Suckling, 2017: 40), o constituir el motor motivacional del jugador para progresar en su partida (Nelson, 2018).

Al explorar los mundos, el jugador puede obtener gran cantidad de información acerca de qué está ocurriendo, cuál es el pasado de esa zona o cómo se relacionan sus habitantes. De esta forma, la historia no se narra como un relato secuenciado, sino que se experimenta mediante estímulos mientras se juega la partida (Codón, 2017).

Dentro de las capas de contenido del mundo, la inclusión de una población resulta esencial para que el jugador encuentre familiaridad en el lugar y se sumerja más en la experiencia (Szymanezyk, Dickinson \& Ducket, 2011: 12). Los personajes del mundo pueden provocar que el jugador olvide 
que se encuentra transitando un mundo ficticio (Černý et al., 2017) y, además, ayudan a conformar la capa biológica del mundo del juego (Plch et al., 2014). Por otra parte, un diseño y una animación de los habitantes que expresen unas necesidades propias aportan situaciones emergentes interesantes que permiten simular con mayor complejidad la vida (Kershner, 2018).

\subsection{Sistemas}

Los sistemas son parte integral del diseño de los mundos abiertos, de tal forma que algunos creadores también los denominan mundos sistémicos (Bergeron, 2017). Se construyen en torno a una inteligencia artificial que relaciona el comportamiento del jugador con los sucesos del mundo, propiciando formas específicas de jugar (Szymanezyk et al., 2011: 3). Por ello, los sistemas conforman una capa de la experiencia de juego que es propia de los videojuegos de mundo abierto (Bergeron, 2017). De esta forma, un sistema es entendido como un mecanismo de la programación y el diseño que puede cumplir varios cometidos. Entre sus funciones, los creadores de videojuegos han explicado dos que consideran esenciales en los videojuegos de mundo abierto. En primer lugar, capacitan al personaje para realizar acciones a lo largo de todo el escenario (England, 2016), por ejemplo, que pueda escalar los edificios de la ciudad en Assassin's Creed (Ubisoft-Montreal, 2007). Pero por otra parte, un sistema permite automatizar elementos del juego para actuar según unos patrones, de tal forma que el mundo de juego reacciona a las acciones del jugador (Canary, 2014). Por ejemplo, la aparición de una ambulancia si algún personaje del mundo ha resultado herido, como ocurre en Saints Row: The Third (Volition, 2011).

La experiencia de juego sistémica, al igual que ocurre con el modelo sandbox, no es exclusiva de los mundos abiertos, puesto que aparece en otros modelos (Laidacker, 2016), pero los mundos abiertos la han adoptado para conseguir que el jugador, haciendo uso de su libertad en el juego, experimente y cree su propia historia. Los autores dejan del lado del jugador la escritura de la historia en los videojuegos de mundo abierto, confiando en que el jugador interactuará con los sistemas (England, 2016) dando lugar a situaciones emergentes, perceptualmente singulares (Short, 2018). Los sistemas funcionan como un mecanismo para construir historias de una manera que recuerda la comparación de la Holocubierta con los videojuegos que planteó Murray (1999) al identificarlos como escenarios digitales procedurales (sistemas de reglas automatizadas de carácter complejo), participativos (relacionado con su naturaleza interactiva), enciclopédicos (en su gestión de la información) y espaciales (que permiten construir entornos en los cuales los jugadores pueden 
navegar). En este caso, los sistemas establecen un conjunto de reglas sobre las historias que se pueden llegar a construir en el juego y articulan historias emergentes dentro del espacio de posibilidades que delimitan.

\section{Propuesta de modelo de análisis}

La complejidad del videojuego como medio se ha ido acrecentando, dejando atrás propuestas simples basadas en la repetición de un grupo reducido de mecánicas para pasar a propuestas de gran profundidad, tanto en jugabilidad como en construcción narrativa. Las experiencias ofrecidas por los títulos modernos presentan grandes diferencias y, por ello, los game studies quieren teorías y modelos de análisis específicos que descubran el verdadero potencial del medio, como apunta Óliver Pérez (2015: 416). A continuación, se ofrece una propuesta de modelo de análisis para videojuegos de mundo abierto que subraya conceptos de su diseño que podrían ser aplicados a cualquier obra del medio, como las mecánicas, los objetivos o la estrategia narrativa, pero también presenta apartados para cuestiones específicas de este modelo, como los sistemas, la variabilidad, la libertad del jugador, la construcción ludonarrativa del mundo o un estudio de los habitantes del mismo.

\subsection{Diseño de la experiencia de juego}

\subsubsection{Objetivo y tema}

Siguiendo la lógica de Chris Crawford (1984), la concepción del título arranca con la definición de dos premisas básicas: el objetivo y el tema (Gómez-García, Navarro-Sierra y Carrillo-Vera, 2019). Por tanto, para poder identificar con exactitud cuál es la experiencia central del título, se debe responder a estas dos cuestiones:

- Objetivo: ¿Qué fantasías y emociones persigue el juego?

- Tema: ¿Cuál es el entorno, momento histórico o contexto en el que se desarrolla la partida?

\subsubsection{Posibilidades de actuación}

La intervención del jugador en el mundo tiene lugar a través de las mecánicas de juego. Para este apartado, se deben describir las mecánicas a través de su vínculo con acciones. Se puede seguir la teoría de Sicart (2008) para identificar cuáles de ellas forman parte del núcleo central del juego y cuáles resultan menos importantes. De esta forma, se deben clasificar de la siguiente forma: centrales, secundarias y otras mecánicas, como se ha señalado en el apartado anterior. 


\subsubsection{Objetivos}

Explicación de qué tipo de objetivos de juego plantea el título como desafíos al jugador. Se debe indicar si son obligatorios para el jugador o no ya que condicionan la construcción de su historia, ya sea por su cumplimiento o por la renuncia a seguirlos como objetivo principal.

\subsubsection{Progresión de la partida}

Debido a las particularidades del diseño, identificar si el título propone una progresión lineal o abierta. En este sentido, se debe indicar si los eventos (bien sean de la trama o los que articule el jugador al margen de la misma) tienen lugar de una forma lineal y predefinida o no; y, por otra parte, si están ligados a un espacio o momento temporal concreto.

\subsubsection{Características de sandbox}

La experiencia de mundo abierto se encuentra ligada al modelo sandbox (Shroff, 2014), por tanto, para entender las posibilidades de experimentación del jugador hay que registrar todo aquel espacio de libertad del que disponga, indicando qué grado de experimentación es posible en el título, si permite diferentes caminos resolutivos para los desafíos y si el jugador puede establecer su propio orden para superarlos.

\subsubsection{Exploración}

En último lugar, es necesario conocer cómo se promueve o favorece la exploración del terreno durante la partida, puesto que la experiencia de estos títulos permite que el jugador conozca el mundo de juego y se familiarice con él.

\subsection{Diseño de la experiencia narrativa}

\subsubsection{Historia del autor}

Descubrir cuál es la historia del autor permite acotar el relato que se pretende contar con el juego, ya que esta, al estar predefinida, siempre tendrá lugar. Además de conocer el relato, también es interesante indicar la estrategia narrativa a utilizar, de tal forma que explicar cómo se estructura (capítulos o secuencias), cómo se cuenta (por ejemplo, durante las misiones o encargos), si hay escenas de vídeo o si se emplea el texto para hacerlo puede resultar útil.

Con respecto a esta historia, es necesario saber si resulta obligatorio para el jugador jugarla, así como estudiar si el desarrollo de la misma está prefijado, si permite toma de decisiones y también si se acaba la partida al terminar la historia propuesta. 


\subsubsection{Historia del jugador}

El jugador puede actuar en el mundo sin la necesidad de seguir el guion preestablecido. Por ello, es necesario detallar qué tipo de historia podría escribir cuando no esté desarrollando la del autor. También resulta de interés precisar cómo afecta la actuación del jugador a la historia del mundo, si tiene consecuencias o si podría ser diferente en cada partida.

\subsubsection{Protagonista de la historia}

El personaje protagonista cumple una función fundamental. Con el objetivo de clasificarlo, se debe descubrir si solo es avatar o si también es dramático (Cuadrado, 2013). En caso de ser dramático, es interesante conocer sus objetivos, motivaciones, necesidades y obstáculos. Además, en algunos casos de estudio es necesario comprobar si evoluciona a lo largo de la historia o no experimenta cambios.

\subsubsection{Historia del mundo}

Puesto que el mundo del videojuego es un espacio ludonarrativo (Planells, 2013), puede transmitir mucha información gracias a la narración ambiental y al diseño de su contenido (Codón, 2017; Connel, 2017; Nitsche, 2008). Para acotar el relato que cuenta el juego, es interesante identificar si existe un marco histórico - si existe un pasado definido- para el entorno.

\subsubsection{El tiempo}

La temporalidad dentro de su relato - atendiendo a si es céntrico o excéntrico (Pérez, 2010) - presenta interés junto a aquellas particularidades que lo hagan asemejarse o diferenciarse del tiempo del mundo real.

\subsubsection{Narrativa emergente}

Dado que el juego pretende representar una simulación del mundo real verosímil, a la vez que permite la experimentación del jugador, resulta útil conocer qué construcciones narrativas toman forma dinámicamente en el mundo de juego como resultado del funcionamiento de sus sistemas y automatizaciones junto a la experiencia de juego que conforma en el jugador y en la experiencia de juego (Gómez-García et al., 2021).

\subsubsection{Consonancia Iudonarrativa}

Como punto de intersección entre el diseño de la experiencia de juego y el diseño narrativo, resulta muy enriquecedor poner en común las mecánicas de juego con la historia para descubrir si se mantiene la consonancia o existe disonancia ludonarrativa (Hocking, 2007). Se puede conseguir identificando 
las mecánicas esenciales (primarias y secundarias) y buscando su relación con el relato.

\subsection{Diseño del mundo de juego}

\subsubsection{Estructura del mundo}

Además de la capa narrativa acerca de la historia del mundo, resulta útil realizar una descripción detallada del entorno de juego. Se debe especificar qué elementos componen el mundo (si son islas, ciudades, calles, continentes o cualquier otra formación), qué límites físicos tiene, qué zonas son transitables y cuáles no, y si existen mecanismos para que el jugador conozca el mundo y domine su tránsito (por ejemplo, letreros, mapas o indicaciones en pantalla).

\subsubsection{Capas del mundo}

Siguiendo las teorías sobre diseños de mundos de videojuegos desde la perspectiva de la geografía (Edwards, 2016), es necesario detallar qué capas de contenido se identifican en el mundo: geofísica, demografía, sistemas e identidades culturales, biosfera, climatología o cualquier otra relevante para el estudio.

\subsubsection{Representaciones del mundo real}

Dentro del objetivo de diseño de representar un mundo verosímil para el jugador, en estos videojuegos se pueden insertar referencias al mundo real. Esto puede manifestarse desde la presencia de personajes del mundo real, entidades, narración de momentos históricos, marcas comerciales u otros elementos que son mostrados de forma fidedigna, hasta representaciones simbólicas o metafóricas de los mismos.

\subsubsection{Sentimiento de pertenencia}

Para que el jugador se sienta un habitante más del lugar, el juego puede utilizar mecanismos, acciones o tomas de decisiones que le vinculen más con el lugar. Esto podría ser la posibilidad de construir su propia casa o formar una familia, entre otras opciones.

\subsubsection{Narración ambiental}

En caso de identificarse narración a través de los entornos en el juego, se deben detallar qué técnicas emplea o qué elementos del mundo desencadenan procesos narrativos en el jugador (Nitsche, 2008). 


\subsubsection{Habitantes}

El análisis de los habitantes del mundo resulta interesante porque pueden ser un elemento transmisor de ideas, además de dotar al mundo de mayor riqueza y dinamismo. Se podría clasificar a los habitantes utilizando la siguiente tabla:

Tabla 1. Clasificación de los habitantes del mundo

\begin{tabular}{|l|l|l|}
\hline Denominación & Actitud & Posición en el mundo \\
\hline & & \\
\hline
\end{tabular}

Fuente: elaboración propia.

Las cuestiones anteriores permiten comprender la posición de los seres (humanos, animales o criaturas) que el jugador se encuentra. La denominación sirve para identificar a la entidad que se está tratando. La experiencia indica que la representación de los habitantes del mundo no siempre se realiza de forma individualizada (personajes con nombre y apellido, por ejemplo), por tanto, es necesario tratar los colectivos como una sola entidad, por ejemplo, ciudadanos, zombis o guardias.

La actitud indica cuál es la relación del habitante (conocido como NPC) con el jugador: aliado, neutral o enemigo (Jungbluth, 2018). Los aliados intentan ayudar al jugador, los neutrales se mantienen pasivos sin involucrarse y los enemigos son aquellos que se configuran como un obstáculo para el jugador.

Por último, la posición en el mundo describe el comportamiento, las motivaciones, objetivos, necesidades o animaciones que manifiesta esa entidad.

\subsubsection{Persistencia de las acciones}

Debido a que el jugador disfruta de una libertad de movimiento y en ocasiones de obra dentro del mundo de juego, se debe estudiar si sus acciones durante los momentos que está desarrollando su partida, al margen de las misiones de juego, modifican el statu quo del mundo o si, por el contrario, no tiene forma de incidir de ese modo en el espacio de juego.

\subsubsection{Reconocimiento del jugador}

Tan interesante resulta estudiar la intervención del jugador en el mundo de juego como descubrir si este manifiesta una respuesta a cambio. Para ello es necesario registrar si el mundo reacciona al jugador o manifiesta signos de ser consciente de su presencia. Esto podría expresarse, por ejemplo, a través de saludos de los habitantes al pasar cerca o, de una forma más integrada en el relato, mediante algún personaje que enuncie las últimas acciones destacadas 
del jugador en el mundo, como ocurre en las emisiones radiofónicas de Fallout 4 (Bethesda-Games-Studios, 2015).

\subsection{Sistemas del mundo}

Los videojuegos de este modelo, al ser concebidos mundos sistémicos (Bergeron, 2017), emplean mecanismos de la programación para mantener el mundo en constante cambio, además de permitir que el jugador desempeñe acciones en cualquier punto del escenario. Por tanto, se deben estudiar por separado los sistemas del mundo y los del jugador.

\subsubsection{Sistemas del mundo}

Es necesario describir todos aquellos sistemas identificados en el mundo, qué controla cada uno y si tiene interacción o influencia sobre otros sistemas. Ejemplos de sistemas del mundo podrían ser el que controla el tráfico mediante semáforos en Grand Theft Auto V (Rockstar-North, 2013) o los cambios atmosféricos en multitud de títulos.

\subsubsection{Sistemas del jugador}

Se deben detallar todos aquellos sistemas identificados en el juego que permitan el desempeño de diversas mecánicas o acciones a lo largo de todo el escenario de juego. Por ejemplo, podrían ser sistemas del jugador los mecanismos de escalada y desplazamiento por el tendido eléctrico en toda la ciudad de Empire City en inFamous (Sucker-Punch, 2009) o mecanismos que sirven para mantener alineación o moralidad dentro del juego, habituales en títulos con componentes de rol.

\subsubsection{Variabilidad y emergencia}

Los sistemas de un mundo abierto ofrecen diversos tipos de situaciones emergentes, no predefinidas en la etapa de diseño. Se debe exponer en qué consiste esa variabilidad, cómo la consigue el juego y cuáles son los resultados de la misma (en relación con la narrativa emergente, anteriormente descrita).

\subsection{Miscelánea}

Dependiendo del objetivo del análisis de un videojuego, pueden ser tenidas en consideración otras cuestiones interesantes para los resultados. Para poder extender las posibilidades de este modelo de análisis al mayor número de títulos de mundo abierto posible adaptándose así a las necesidades de cada estudio, puede ser interesante incluir un último apartado en el que se registren aquellos elementos de diseño que no han podido incluirse en los anteriores apartados, como pudieran ser la posición de la cámara, la duración 
de la partida u otras cuestiones que, según el caso, tengan relevancia y se consideren oportunas.

\section{Resultados de análisis}

Para comprobar la eficacia del modelo de análisis, se ha aplicado al videojuego Death Stranding (Kojima-Productions, 2019), cuya propuesta de juego se ajusta a la experiencia de mundo abierto. Los resultados se ofrecen en los siguientes epígrafes, ordenados en bloques temáticos que permiten describir mejor su diseño ludonarrativo.

\subsection{La conexión como acción vertebradora}

Death Stranding (Kojima-Productions, 2019) es una obra que habla sobre «conectar» en una época en la que las nuevas tecnologías ganan terreno como medio de comunicación frente al tradicional encuentro cara a cara. El análisis de significación del conjunto de reglas, mecánicas y objetivos indica que la conexión es la experiencia primordial de su partida.

El objetivo y tema que plantea es la reconexión de Estados Unidos en un futuro postapocalíptico. El jugador, a través de Sam Porter, debe actuar como mensajero para transportar paquetes e información que permitan crear una red similar a Internet. En ese contexto, Estados Unidos ha resultado destruido tras lo que se plantea como un ataque terrorista, originando una desconexión de las ciudades y los puntos industriales entre sí. El viaje de Sam consiste en recorrer el país de costa a costa para conectar a la red todos los núcleos poblacionales supervivientes, reconstruyendo así los vínculos sociales, unificando la producción y restaurando el gobierno central en todo el territorio.

La conexión, por tanto, se convierte en el tema central de toda la experiencia. Argumentalmente, las acciones de Sam permiten que se recuperen los vínculos en una sociedad que se encuentra aislada, pero las mecánicas de juego también insisten en la misma idea. Desde una visión más evidente, el jugador juega a conectar el terreno. El transporte de los paquetes le obliga a superar terrenos de difícil acceso y debe utilizar herramientas como escaleras, cuerdas de escalada o puentes.

Por otra parte, el protagonista también tiene que mantener una relación casi paternofilial con un bebé que lleva en una incubadora portátil. Este reacciona a algunas acciones del jugador mostrando su felicidad o miedo, pero además tiene asociadas unas mecánicas de interacción, como acunarle o hablarle para que se tranquilice, como puede observarse en la Figura 1, que llevan a mejorar el vínculo. 


\section{Imagen 1. Captura de juego de Death Stranding}

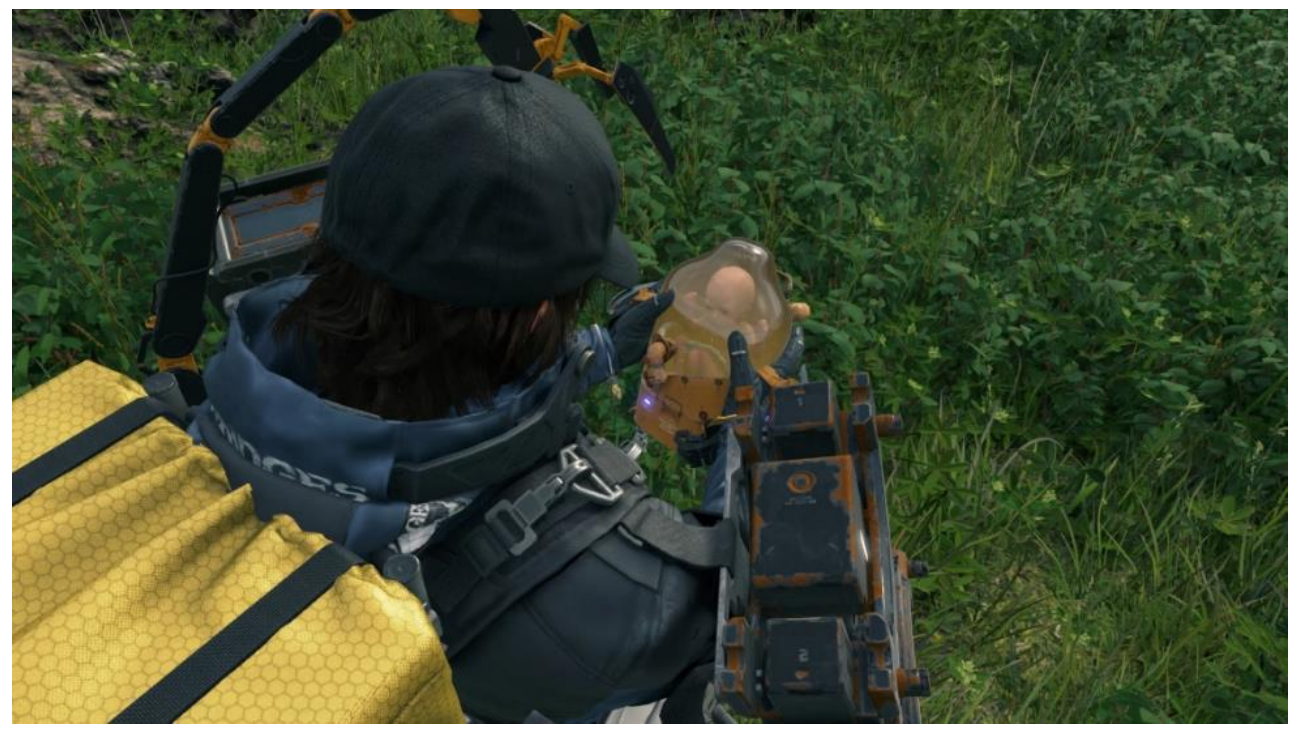

Fuente: Death Stranding (Kojima-Productions, 2019).

Esta conexión también tiene una dimensión simbólica en las acciones del jugador al transportar los paquetes. Las mecánicas permiten agarrar, sostener, soltar y lanzar objetos. En caso de utilizar un mando, el jugador emplea un botón del lado derecho para que Sam haga uso de su brazo derecho y un botón del lado izquierdo para que use el brazo izquierdo. Al pulsar uno de ellos, Sam agarra objetos con la mano correspondiente y, en caso de no tener nada al alcance, sujeta con fuerza su mochila para reequilibrar el peso del cuerpo; cuando suelta el botón, se libera la acción de esa mano. De esta forma se crea una cadena de conexiones: el jugador conecta con el protagonista a través de los botones de una forma intuitiva, y este lo hace con los objetos el mundo.

\subsection{Experiencia de juego compleja}

La primera conclusión de las mecánicas de juego identificadas es que se trata de un título complejo, ya que se han registrado hasta 38 verbos de acción diferentes. Su análisis indica que la mayoría de las esenciales se corresponden con acciones físicas relacionadas con el cuerpo del personaje. Partiendo de la base de que la mayoría de los objetivos planteados por el juego consisten en transportar paquetes, cuyo principal desafío es el enfrentamiento del hombre con el terreno natural, mantiene consonancia ludonarrativa con las acciones primarias y secundarias para superar desafíos que estén relacionados con el desplazamiento (andar, correr, escalar, conducir o equilibrar el cuerpo, entre 
otros), pero también otros relacionadas con atender a necesidades biológicas, como dormir, beber, descansar para recuperar energía u orinar.

\subsection{Mundo estable, aunque maleable}

El mundo postapocalíptico de Death Stranding lo conforman dos grandes terrenos, de diferente magnitud, separados por una masa de agua, que representan a Estados Unidos. El jugador puede desplazarse por toda la amplitud de sus zonas agrestes empleando herramientas o vehículos. La Figura 2 muestra cómo el jugador puede escalar cualquier montaña para plantear su estrategia $-o$ ruta - a seguir.

\section{Imagen 2. Captura de Death Stranding}

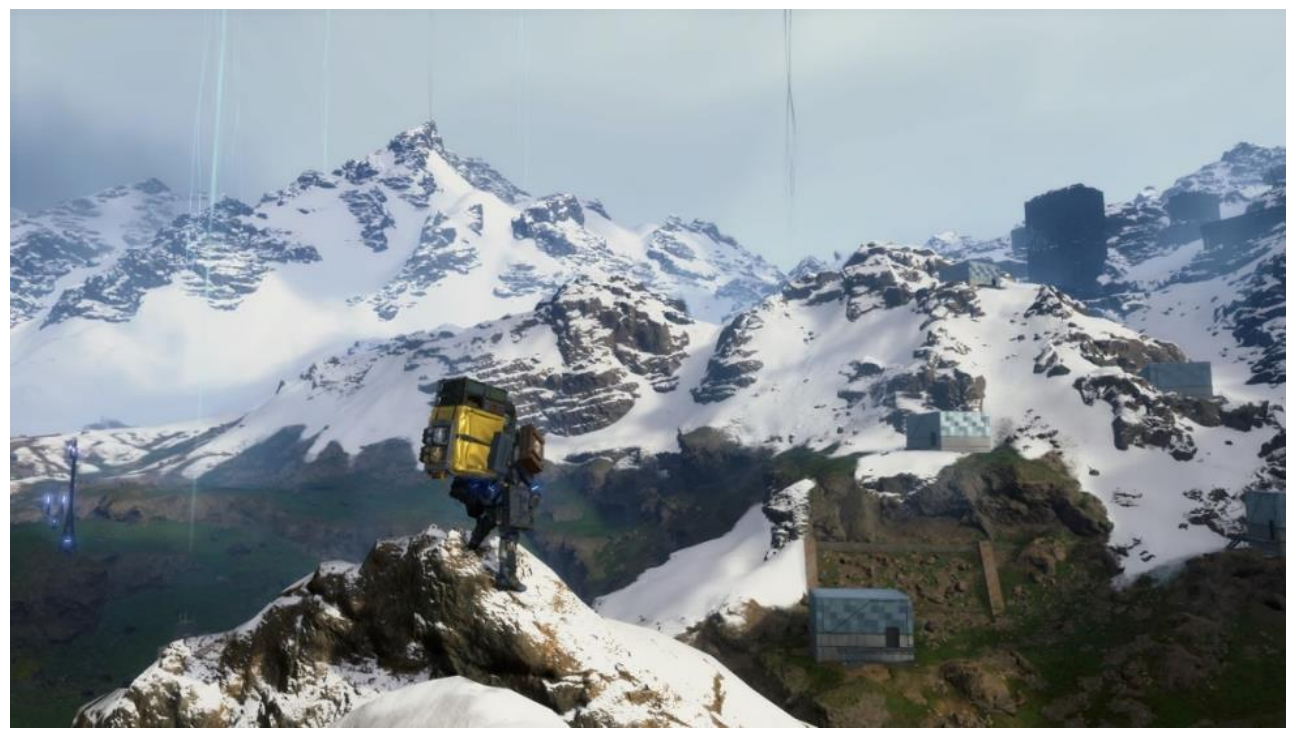

Fuente: Death Stranding (Kojima-Productions, 2019).

El diseño del mundo se encuentra firmemente sujeto bajo la mano del creador, quien ha configurado el gran terreno como la suma de diferentes espacios y biomas (montaña nevada, campiña, desierto o bosques) y ha establecido qué ocurrirá en cada uno de ellos.

La ecología de los NPC plantea tres tipos principales. En primer lugar, los habitantes de las ciudades, que solo aparecen en escenas de vídeo y ayudan al jugador. Por otra parte, se encuentran los saqueadores y seres sobrenaturales, ambos hostiles, que están establecidos de forma permanente en unas localizaciones claves para el tránsito, funcionando como un obstáculo agresivo 
para el jugador cuando pretenda atravesarlas. En ningún caso se relacionan o interactúan entre sí los personajes de cada tipo.

Los sistemas del mundo en este caso se vinculan principalmente a la afinidad del protagonista con determinadas localizaciones o personajes, dando acceso o no a algunas funciones, aunque también se ha detectado que existe un sistema encargado de generar paquetes aleatoriamente por el mapa que el jugador puede recoger y entregar en diversos puntos para obtener recursos. Entre los sistemas del jugador, destaca su vínculo cuantificado con el bebé, así como los mecanismos que permiten la conducción y la escalada en cualquier lugar del mapa.

Una planificación de los desafíos con tan poca variabilidad, ya que los enemigos se encuentran asociados a una zona concreta del mapa y no se producen encuentros aleatorios, origina menos emergencia en la partida, puesto que superarlos desemboca en situaciones similares en cada intento. En este caso, la variabilidad se presenta en el encuentro fortuito de objetos, susceptibles de convertirse en objetivos para jugador.

No obstante, el diseño del mundo sí que deja espacio para la modificación del terreno. En concreto, el jugador puede restaurar carreteras e insertar estructuras y herramientas para mejorar su tránsito. Dentro de las posibilidades que se ofrecen se encuentran puentes, estaciones de reparación de paquetes, tirolinas y salas de descanso, además de elementos de menor tamaño como escaleras o cuerdas de escalada. Estos artefactos se mantienen en el mundo de forma permanente hasta que sean eliminados manualmente o hasta que el desgaste y la falta de mantenimiento los deje en un estado inservible.

\subsection{Experiencia multijugador asíncrona}

Si el jugador se conecta a Internet, accede a un modo multijugador asíncrono que conecta su mundo de juego con el de otros jugadores. Esto provoca que, una vez descubiertas las zonas del mapa, aparezcan estructuras creadas por otros jugadores, como puentes o estaciones de descanso. Esta funcionalidad se encuentra en sintonía con el relato, ya que se interpreta que esos artefactos han sido colocados por otros portadores que han pasado antes por el lugar. Además, puede crear vínculos con los dueños de las estructuras otorgándoles puntos como gratitud al activar el comando «Me gusta» sobre dichas creaciones, de forma similar a como funciona una red social.

\subsection{Narrativa y progreso en la partida}

El diseño de la partida permite que el jugador aplace la superación de objetivos y dedique tiempo a satisfacer otras motivaciones, como explorar el mundo de 
juego o cumplir tareas secundarias no esenciales para la trama. Esta decisión provoca que la narración de la historia del autor (Van-Lierop, 2018) se encuentra asociada a la superación de objetivos. Cuando el jugador entrega algunos paquetes esenciales para el argumento, se desencadenan cinemáticas en las que se narran los principales eventos de la historia. Esta técnica narrativa tiene una gran relevancia en el tramo final de la partida, en el que se desenlazan los acontecimientos a través de una serie de escenas cinematográficas que superan la hora de duración.

Durante el transcurso de la partida, la narrativa se adapta al ritmo del jugador. Cuando los acontecimientos requieran la intervención de algún personaje, recibe una llamada telefónica en la que le transmiten la información necesaria. Por otra parte, el juego entrega textos escritos que narran historias del mundo y sus personajes de forma escalonada para que el jugador los lea cuando quiera expandir sus conocimientos.

\section{Conclusiones}

El modelo de videojuegos de mundo abierto plantea un diseño con particularidades propias que le diferencia de otros con desarrollo más clásico, como pueden ser los de progresión lineal. Su partida, que descansa sobre premisas relacionadas con la libertad de maniobra del jugador, el fomento de la exploración del mundo de juego y la sistematización de los sucesos en el juego, origina nuevas formas de jugar.

Un modelo de análisis específico, que atienda a estas características de diseño, favorece obtener una visión holística de estos títulos, complejos en su experiencia de juego. Los apartados del modelo, basados en parcelas temáticas, resultan útiles a la hora de relacionar las características de cada obra con su objetivo de diseño. Un estudio con herramientas específicas permite llegar a conclusiones más exactas que otras técnicas de análisis que no tengan en cuenta la naturaleza de las obras analizadas.

El caso de Death Stranding ha servido para descubrir una propuesta lúdica basada en la creación de vínculos para progresar en la partida dentro de un mundo que incita a la exploración del jugador y consiente la modificación de su aspecto. En el plano narrativo, se ha comprobado que el relato concede pausas en su desarrollo para que el jugador realice otras actividades al margen de las misiones principales. De esta forma, la narración avanza significativamente al superar objetivos, aunque de forma constante se obtienen flujos de información mediante otras técnicas adaptadas al ritmo del jugador, como conversaciones telefónicas o textos escritos de libre consulta. 


\section{Referencias bibliográficas}

ARAnda, D., GómeZ, S., NAVARRo-Remesal, V., \& Planells, A. J. (2015). Game \& Play. Diseño y análisis del juego, el jugador y el sistema lúdico. Uocp.Comunicación

BERGERON, P. (2017). 360 Approach for Open World Mission Design in Far Cry and Assassin's Creed. Game Developers Conference 2016.

https: / / tinyurl.com/yyye3prk

BERMEJO, J. (2013). El videojuego: Cibertexto y Cajón de arena. 25-37.

BETHESDA-GAMES-STUDIOS. (2015). Fallout 4.

CANARY, A. (2014). Free Range AI: Creating Compelling Characters for Open World Games Title. Game Developers Conference 2014.

https: / / tinyurl.com/r6tjbnb8

ČERNÝ, M., PLCH, T., MARKO, M., GEMROT, J., ONDRÁČEK, P., \& BROM, C. (2017). Using behavior objects to manage complexity in virtual worlds. IEEE Transactions on Computational Intelligence and AI in Games, 9(2), 166-180.

https://doi.org/10.1109/TCIAIG.2016.2528499

ClausSEn, A. (2019). Unpopular Opinion: All Narrative is Linear. Game Developers Conference 2017 - Game Narrative Summit.

https://tinyurl.com/979njk75

CoDón, Á. (2017). Narrativa in-game y narrativa ambiental. En Curso de diseño narrativo y guión de videojuegos. https: //tinyurl.com/f4en9e4y

Connel, J. (2017). Ghost of Tsushima Interview: Details on Sucker Punch's Next Open World Adventure / PS4. YouTube: PlayStation.

https: / / tinyurl.com/a88t4c9v

CRAWFORD, C. (1984). The Art of Computer Game Design. Washington State University. https://doi.org/10.1016/S0920-5632(03)90636-0

CRAWFORD, C. (2005). Chris Crawford on Interactive Storytelling. New Riders Publishing.

CuADRADO, A. (2013). Acciones y emoción: un estudio de la jugabilidad en Heavy Rain. En Homo Videoludens 2.0. De Pacman a la gamification (pp. 151-176). Laboratori de Mitjans Interactius. Universitat de Barcelona. DMA-DESIGN. (2001). Grand Theft Auto III.

EDWARDS, K. (2016). A Geographer's Guide to Building Game Worlds. Game Developers Conference Europe 2016. https: //tinyurl.com/4astjwxh

ENGLAND, L. (2016). Sunset Overdrive: Transitioning from Linear to Open World Design. Game Developers Conference 2015.

https: //tinyurl.com/5ab4yjw9

FERNÁNDEZ-VARA, C. (2009). The tribulations of adventure games: Integrating story into simulation throuhg performance. Georgia Instute of Technology. 
FRASCA, G. (1999). LUDOLOGY MEETS NARRATOLOGY: Similitude and differences between (video) games and Ludology. Parnasso\#3, 1-11. https: / / tinyurl.com/y6wadbwu

Garrelts, N. (2006). The Meaning and Culture of Grand Theft Auto: Critical Essays. https: / / tinyurl.com/cu29p5b3

GómEZ-GarcíA, S., NAVARro-SiERrA, N., \& CARrillo-VERA, J.-A. (2019). De la lucha al testimonio. Las representaciones audiovisuales e interactivas del cáncer en los videojuegos. Interfaçe-Comunicação, Saúde, Educacão, 23.

GómeZ-García, S., PAZ-REbollo, M. A., \& CABEZA-SAN-DEOGraCiaS, J. (2021). Newsgames against hate speech in the refugee crisis [Newsgames frente a los discursos del odio en la crisis de los refugiados]. Comunicar, 67, 123-

133. https: / / doi.org/10.3916/C67-2021-10

HockING, C. (2007). Ludonarrative Dissonance in Bioshock.

https: / / tinyurl.com/4x6r96dh

ISBISTER, K. (2017). How games muve us. Emotion by design. MIT Press.

JungBluTH, M. (2018). Establishing an Ecology for NPCs. Game Developers Conference 2014. https: / / tinyurl.com/y3k83wu6

JUUL, J. (2005). Half-Real: Video Games between Real rules and Fictional Worlds. KELLE, U. (2010). The development of categories: Different approaches in grounded theory. In A. Bryant \& K. Charmaz (Eds.), The Sage handbook of grounded theory (pp. 191-213). Sage Publications.

KERSHNER, C. J. (2018). The Lives of Others: How NPCs Can Increase Player Empathy. Game Developers Conference 2016 - Game Narrative Summit. https: / / tinyurl.com/t2dyrtud

KoJIMA-PRoduCtions. (2019). Death Stranding.

LAIDACKER, A. (2016). GCAP 2016: Systems Are Everywhere - Aleissia Laidacker. Game Connect Asia Pacific 2016: The Soulders of Giants. https: / / tinyurl.com/f8ww4pks

LAUREL, B. (2013). Computers as theatre. Addison-Wesley.

Mikkelsen, J., \& Podenphant, M. (2019). Level Design in Hitman: Guiding Players in a Non-Linear Sandbox. Game Developers Conference Europe 2016. https: / /tinyurl.com/jxmpdus6

Min, W., MotT, B., Rowe, J., LiU, B., \& LESTER, J. (2016). Player Goal Recognition in Open-World Digital Games with Long Short-Term Memory Networks. IJCAI.

MunCY, J. (2015). Open-World Games Are Changing the Way We Play. Wired. https: / /tinyurl.com/37s5xp6m

MURRAY, J. (1999). Hamlet en la holocubierta: el futuro de la narrativa en el ciberespacio. Paidós. 
NAVARRo, V. (2013). Libertad dirigida: análisis formal del videojuego como sistema, su estructura y su avataridad. https: //doi.org/T. 615-2013

NELSON, R. (2018). Red Dead Redemption 2 - exclusive Rockstar Games interview. BBCRadio1. https: //tinyurl.com/63kuttvc

NinTEndo-EAD. (2012). Animal Crossing: New Leaf.

NiTSCHE, M. (2008). Video Game Spaces: Image, Play and Structure in 3D Game Worlds. Video Game Spaces: Image, Play, and Structure in 3D Game Worlds, 1-24. https://doi.org/10.4018/jgcms.2009091505

PARKER, J. (2015). Introduction to Game Development using Processing. Mercury Learning and Information.

PÉREZ, Ó. (2010). Análisis de la significación del videojuego. Fundamentos teóricos del juego, el mundo narrativo y la enunciación interactiva como perspectivas de estudio del discurso (Tesis Doctoral, Universidad Pompeu Fabra).

PÉREZ, Ó. (2015). The Social Discourse of Civdeo Games. Analysis Model and Case Study: GTA IV. Games and Culture, 10(5), 415-437.

Planells, A. (2013). Los videojuegos como mundos ludoficcionales. Una aproximación semántico-pragmática a su estructura y significación.

Plch, T., Marko, M., OndráčEK, P., ČERnÝ, M., GEMrot, J., \& Brom, C. (2014).

Modular behavior trees: Language for fast ai in open-world video games.

Frontiers in Artificial Intelligence and Applications, 263, 1209-1210.

https: / / doi.org/10.3233/978-1-61499-419-0-1209

RoCKSTAR-NorTH. (2013). Grand Theft Auto V.

SHORT, T. X. (2018). Writing Modular Characters for System-Driven Games.

Game Developer Conference 2018. https: / / tinyurl.com/3a6cp7vx

ShrofF, J. (2014). Free Range AI: Creating Compelling Characters for Open

World Games Title. Game Developer Conference 2014.

https: / / tinyurl.com/r6tjbnb8

SICART, M. (2008). Defining Game Mechanics. Game Studies, Volume 8(Issue 2).

https: / / tinyurl.com/6tfpu7hb

Solo, M. (2019). Historia del Mundo Abierto: Expandiendo la frontera digital.

https: / / tinyurl.com/28ptwsk9

SQUIRE, K. (2008). Open-ended videogames: A model for developing learning

for the interactive Age. In The ecology of games: Connecting youth, games

and learning (pp. 167-198). The MIT Press.

https: / / doi.org/10.1162/dmal.9780262693646.167

SUCKER-PUNCH. (2009). Infamous.

SUCKER-PUNCH. (2020). Ghost of Tsushima. 
SZYMANEZYK, O., DiCKInSON, P., \& DUCKETT, T. (2011). From Individual

Characters to Large Crowds: Augmenting the Believability of Open-World Games through Exploring Social Emotion in Pedestrian Groups. DiGRA 2011: Think Design Play. https: / / tinyurl.com/2djsc2s2

Todorov, T. (2006). Las categorías del relato literario. In Análisis Estructural del Relato (pp. 155-192).

TREMBLAY, K. (2020). Storytelling with Verbs: Integrating Gameplay and Narrative. Game Developer Conference 2020.

https: / / tinyurl.com/tkby6hd7

UBISOFT-MONTREAL. (2007). Assassin's Creed.

VAN-LIEROP, R. (2018). A Long Dark Road: Blending Player and Authored Story in a Sandbox Survival Game. Game Developers Conference 2018.

https: / / tinyurl.com/tb4src5j

Volition. (2011). Saints Row: The Third.

Walton, M., \& Suckling, M. (2017). Video Game Writing. From Macro to Micro. Mercury Learning and Information.

ZAGAL, J., \& MATEAS, M. (2007). Temporal Frames: A Unifying Framework for the Analysis of Game. Situated Play.

Zimmerman, E., \& SALEN, K. (2003). Rules of Play: Game Design Fundamentals. MIT Press. 